\title{
Uniqueness of positive solutions to a class of semilinear elliptic equations
}

\author{
Chunming Li and Yong Zhou*
}

\author{
* Correspondence: \\ yzhoumath@zjnu.edu.cn \\ Department of Mathematics, \\ Zhejiang Normal University, Jinhua \\ 321004, Zhejiang, PR China
}

\begin{abstract}
In this article, we consider the uniqueness of positive radial solutions to the Dirichlet boundary value problem

$$
\begin{array}{ll}
\Delta u+f(|x|, u)+g(|x|) x \cdot \nabla u=0, & x \in \Omega, \\
u=0, & x \in \partial \Omega,
\end{array}
$$

where $\Omega$ denotes an annulus in $\mathbb{R}^{n}(n \geq 3)$. The uniqueness criterion is established by applying shooting method.
\end{abstract}

Keywords: positive solution, semilinear elliptic equation, uniqueness

\section{Introduction}

This article is concerned with the positive radial solutions to a class of semilinear elliptic equations

$$
\begin{array}{ll}
\Delta u+f(|x|, u)+g(|x|) x \cdot \nabla u=0, & x \in \Omega, \\
u=0, & x \in \partial \Omega,
\end{array}
$$

where $\Omega:=\left\{x\left|x \in \mathbb{R}^{n}, a<\right| x \mid<b\right\}, a$ and $b$ are positive real numbers, $f \in C^{1}((0,+$ $\infty) \times[0,+\infty))$ and $g:[0,+\infty) \rightarrow \mathbb{R}$ is differentiable. Equation 1.1 describes stationary states for many reaction-diffusion equations. The absence of positive solutions to the elliptic equations also means that the existing solutions oscillate, which is also important information in applications.

In recent years, there is a widespread concern over the positive solutions to the Dirichlet boundary value problem (1.1) when $g(|x|)=0$, i.e.,

$$
\begin{array}{ll}
\Delta u+\mid f(|x|, u)=0, & u>0 \text { in } \Omega, \\
u=0, & x \in \partial \Omega .
\end{array}
$$

When the nonlinear term just depends on $u$, the uniqueness of (1.2) has been exhaustively studied (see [1-6]). In 1985, the uniqueness of (1.2) was discussed in different domains by $\mathrm{Ni}$ and Nussbaum [7] to the case when $f$ depends on $|x|$ and $u, f(\mid$ $x \mid, u)>0$ and $f(|x|, u)$ satisfies some growth conditions. Erbe and Tang [8] presented a new uniqueness criterion using a shooting method and Sturm comparison theorem.

So far it seems that nobody considers the uniqueness to problem (1.1). Inspired by the above articles, the aim of the present article is to establish some simple criteria for the uniqueness of positive radial solutions to problem (1.1). Obviously, what we investigate in this article has a more general form than (1.2). Although due to technical

(c) $2011 \mathrm{Li}$ and Zhou; licensee Springer. This is an Open Access article distributed under the terms of the Creative Commons Attribution License (http://creativecommons.org/licenses/by/2.0), which permits unrestricted use, distribution, and reproduction in any medium, provided the original work is properly cited. 
reasons, when $g(|x|)=0$ it does not hold in this article, there exist many other $g(|x|)$ which satisfy our main result.

We now conclude this introduction by outlining the rest of this article. In Section 2, we will show the existence and uniqueness of positive solutions of the initial problem

$$
\begin{aligned}
& u^{\prime \prime}+h(t) u^{\prime}+f(t, u)=0, \\
& u(a)=0, u^{\prime}(a)=\alpha,
\end{aligned}
$$

where $\alpha>0$. Our method is the Schauder-Tikhonov fixed point theory. The existence and uniqueness of this initial problem is important to prove our main result. In Section 3, we will give the proof of our main result, i.e., show the uniqueness of positive solutions to Equation 1.1, using a shooting method and Sturm theorem.

\section{Preliminaries}

To consider the positive radial solutions of Equation 1.1, it is reasonable to investigate the corresponding radial equation

$$
u^{\prime \prime}+\frac{n-1}{t} u^{\prime}+f(t, u)+\operatorname{tg}(t) u^{\prime}=0,
$$

where $t=|x|$. For giving a proof of uniqueness of problem (1.1), let us consider the initial problem

$$
\begin{array}{ll}
u^{\prime \prime}+h(t) u^{\prime}+f(t, u)=0, & t \in[a, b], \\
u(a)=0, & u^{\prime}(a)=\alpha
\end{array}
$$

where $\alpha>0, h(t)=\frac{n-1}{t}+\operatorname{tg}(t)$. We shall show that problem (2.1) has a unique positive solution. By a solution to problem (1.2), we mean $u \in C^{2}$ and $u>0$ for all $t \in$ $(a, b)$. First of all, we give a well-known lemma.

Lemma 2.1 (The Schauder-Tikhonov fixed point theorem [9]). Let $\times$ be a Banach space and $K \subset X$ be a nonempty, closed, bounded and convex set. If the operator $T: K$ $\rightarrow X$ continuously maps $K$ into itself and $T(K)$ is relatively compact in $X$, then $T$ has a fixed point $x \in K$.

Theorem 2.1 If there exist $m$ and $M$, such that $0<m \leq u \leq M$ for $u \in C([a, b],(0$, $\infty)$ ) and

$$
m \leq \int_{a}^{t} e^{-\int_{a}^{s} h(\xi) d \xi}\left(\alpha-\int_{a}^{s} e^{\int_{a}^{\xi} h(r) d r} f(\xi, u(\xi)) d \xi\right) d s M, \quad a<t<b .
$$

Then, Equation 2.1 has a unique positive solution.

Proof. We assume that

$$
X=\left\{u \in C([a, b],(0, \infty)): \sup _{a \leq t \leq b}|u(t)|<\infty\right\},
$$

endowed with the supremum norm $\|u\|=\sup _{a \leq t \leq b}|u(t)|$. Let

$$
K=\{u \in X: m \leq u(t) \leq M, t \in(a, b)\} .
$$

Define the operator $T: K \rightarrow X$, by

$$
(T u)(t)=\int_{a}^{t} e^{-\int_{a}^{s} h(\xi) d \xi}\left(\alpha-\int_{a}^{s} e^{\int_{a}^{\xi} h(r) d r} f(\xi, u) d \xi\right) d s, \quad a<t<b .
$$


We shall apply the Schauder-Tikhonov theorem to prove that there exists a fixed point $u(t)$, which is a positive solution of problem (2.1), for the operator $T$ in the nonempty closed convex set $K$.

We shall do it by several steps as follows:

Step 1: Check that $T: K \rightarrow K$ is well defined. Obviously, by (2.2), we have

$$
m \leq T u(t) \leq M, \quad u(t) \in K,
$$

thus $T: K \rightarrow K$ is well defined.

Step 2: Verify that $T: K \rightarrow K$ is continuous. Note that $h(t), f(t, u)$ are continuous, they are integrable on $[a, b]$, there exists a constant $M_{1}$ such that

$$
0<\int_{a}^{t} e^{-\int_{a}^{s} h(\xi) d \xi}\left(\int_{a}^{s} e^{\int_{a}^{\xi} h(r) d r} d \xi\right) d s \leq M_{1,} \quad t \in[a, b] .
$$

The function $f(t, u)$ is continuous, thus for $\forall \varepsilon>0$, there exists $\delta>0$ such that for any $u(t), v(t) \in K$ with $\|u-v\| \leq \delta$,

$$
|f(t, u)-f(t, v)| \leq \frac{\varepsilon}{M_{1}} .
$$

From this, it follows that

$$
\begin{aligned}
|T u(t)-T v(t)| & =\mid \int_{a}^{t} e^{\int_{a}^{s} h(\xi) d \xi}\left(\alpha-\int_{a}^{s} e^{\int_{a}^{\xi} h(r) d r} f(\xi, u(\xi)) d \xi\right) d s \\
& -\int_{a}^{t} e^{-\int_{a}^{s} h(\xi) d \xi}\left(\alpha-\int_{a}^{s} e^{\int_{a}^{\xi} h(r) d r} f(\xi, v(\xi)) d \xi\right) d s \mid \\
& \leq \int_{a}^{t} e^{-\int_{a}^{s} h(\xi) d \xi}\left(\int_{a}^{s} e^{\int_{a}^{\xi} h(r) d r}|f(\xi, u)-f(\xi, v(\xi))| d \xi\right) d s \\
& \leq \varepsilon .
\end{aligned}
$$

Thus, $T$ is continuous on $K$.

Step 3: We check that $T(K)$ is relatively compact in $X$.

Since $T K \subset K, T K$ is uniformly bounded. Now, verify that $T K$ is equicontinuous. Let $u \in K$, then we have

$$
(T u)^{\prime}(t)=e^{-\int_{a}^{t} h(s) d s}\left(\alpha-\int_{a}^{t} e^{\int_{a}^{s} h(r) d r} f(s, u) d s\right) .
$$

Similar to (2.3), there exists a constant $M_{2}$ such that

$$
\left|(T u)^{\prime}(t)\right| \leq M_{2}, \quad a<t<b .
$$

Take a sequence $\left\{u_{n}\right\} \subset K$, by the mean value theorem, we have

$$
\left|\left(T u_{n}\right)\left(t_{1}\right)-\left(T u_{n}\right)\left(t_{2}\right)\right| \leq M_{2}\left|t_{1}-t_{2}\right|, \quad a<t<b .
$$

Thus, $T K$ is equicontinuous. Arzela-Ascoli theorem [9] implies $T K$ is relatively compact. Now, we have verified that $T: K \rightarrow K$ satisfies all assumptions of the SchauderTikhonov theorem. Thus, there exists a fixed point $u$ which is a positive solution of problem (2.1).

Now, we are in a position to prove the uniqueness of problem (2.1). The proof of the uniqueness of solution is based on the work of [10]. Suppose that $u$ and $v$ are two different solutions of problem (2.1), then the function 


$$
\omega=u^{\prime}-v^{\prime}
$$

is a solution of Cauchy problem

$$
\omega^{\prime}+h(t) \omega^{\prime}=\Psi(t), \quad \omega(a)=0,
$$

where $\psi=f(t, v)-f(t, u)$. It follows that

$$
\omega=e^{-\int_{a}^{t} h(s) d s} \int_{a}^{t} e^{\int_{a}^{r} h(s) d s} \Psi(r) d r .
$$

Hence, we have

$$
\begin{aligned}
|\omega(t)| & \leq e^{-\int_{a}^{t} h(s) d s} \int_{a}^{t} e^{\int_{a}^{r} h(s) d s}|\Psi(r)| d r \\
& \leq M_{3} \sup _{a \leq t \leq b}|\Psi(t)|
\end{aligned}
$$

where $M_{3}$ is a constant, such that

$$
0<e^{-\int_{a}^{t} h(s) d s} \int_{a}^{t} e^{\int_{a}^{r} h(s) d s} d r \leq M_{3}, \quad t \in[a, b] .
$$

On the other hand, since the function $f(t, u)$ is Hölder continuous with respect to the second variable on $(0,+\infty)$, we obtain, for appropriate values $t_{0}, L>0$,

$$
\begin{aligned}
|\Psi(t)| & \leq L|u(t)-v(t)| \\
& \leq L \int_{a}^{t}\left|u^{\prime}(s)-v^{\prime}(s)\right| d s \\
& \leq L \int_{a}^{t}|\omega(s)| d s, \quad t \in\left(a, t_{0}\right] .
\end{aligned}
$$

From this, we have $|\omega(t)| \leq M_{3} L \int_{a}^{t}|\omega(s)| d s$ for $t \leq t_{0}$. It now follows from Gronwall's inequality that $\omega \equiv 0$ for $a<t \leq t_{0}$, consequently $u^{\prime} \equiv v^{\prime}$ for $t \leq t_{0}$. We find $u(t) \equiv$ $v(t)$ for all $t \in\left(a, t_{0}\right]$. With the initial point $t_{0}$ replace by $\rho>t_{0}$, for an appropriate value $\rho$, the same proof can be reapplied as often as necessary to give uniqueness of any continuation of the solution whose values lie in $(a, b)$. The proof is complete.

\section{Uniqueness}

Theorem 3.1 Assume that $h(t)$ and $f(t, u)$ for $a<t<b, u(t)>0$, satisfy inequality (2.2) and

$$
\begin{aligned}
& (F 1) f(t, 0) \equiv 0, u f_{u}(t, u)>f(t, u)>0, \\
& (F 2) f_{1}(t, u) \geq 0, h(t) v(t)+v^{\prime} \geq 0, \\
& (F 3) h^{\prime}(t) \geq 0
\end{aligned}
$$

where $v(t)=\int_{a}^{t} e^{-\int_{a}^{r} h(s) d s} d r$, then problem (1.1) has at most one positive radial solution.

Example 3.1 For the equation

$$
\Delta u+\frac{A}{|x|^{2}} x \cdot \nabla u+u^{2}=0, \quad x \in \Omega,
$$


where $-n-1 \leq A \leq-n+1, \Omega:=\left\{x \in \mathbb{R}^{n}\left|\frac{1}{2}<\right| x \mid<1\right\}, n \geq 3$. Let $t=|x|$, then

$$
h(t)=\frac{A+n-1}{t}, \quad v(t)=\int_{\frac{1}{2}}^{t} e^{-\int_{1}^{r} \frac{A+n-1}{s} d s} d r .
$$

A straightforward computation yields

$$
h^{\prime}(t)=-\frac{A+n-1}{t^{2}} \geq 0
$$

and

$$
h(t) v(t)+v^{\prime}(t)=e^{-(A+n-1)}\left(t(A+n+1)-\frac{A+n-1}{4 t}\right) \geq 0, \quad t \in\left(\frac{1}{2}, 1\right) .
$$

Therefore, Theorem 3.1 ensures that there exists at most one positive radial solution. Before proving our main result, we will do some preliminaries and give some useful lemmas.

Let $u(t, \alpha)$ denote the unique solution of Equation 2.1. If $\alpha>0$, then the solution $u(t$, $\alpha)$ is positive for $t$ slightly larger than $a$. When it vanishes in $(a, b)$, we define $b(\alpha)$ to be the first zero of $u(t, \alpha)$. More precisely, $b(\alpha)$ is a function of $\alpha$ which has the property that $u(t, \alpha)>0$ for $t \in(a, b(\alpha))$ and $u(b(\alpha), \alpha)=0$. Let $N$ denote the set of $\alpha>0$ for which the solution $u(t, \alpha)$ has a finite zero $b(\alpha)$. The variation of $u(t, \alpha)$ is defined by

$$
\phi(t, \alpha)=\frac{\partial u(t, \alpha)}{\partial \alpha}
$$

and satisfies

$$
\phi^{\prime \prime}+h(t) \phi^{\prime}+f_{u}(t, u) \phi=0, \quad \phi(a, \alpha)=0, \quad \phi(a, \alpha)=1 .
$$

Let $L$ be the linear operator given by

$$
L(\phi(t, \alpha))=\phi^{\prime \prime}+h(t) \phi^{\prime}+f_{u}(t, u) \phi, \quad a \leq t \leq b(\alpha) .
$$

By (2.4), it is easy to show that $u(t, \alpha)$ has a unique critical point $c(\alpha)$ in $(a, b(\alpha))$, and at this point, $u(t, \alpha)$ obtains a local maximum value.

Lemma 3.1 Assume that (F2) holds, then $\varphi(t, \alpha)>0$ for all $t \in(a, c(\alpha))$.

Proof. We introduce a function

$$
Q(t, \alpha)=\frac{v(t)}{v^{\prime}(t)} u^{\prime}(t, \alpha) \geq 0, \quad a \leq t \leq c(\alpha),
$$

where

$$
v(t)=\int_{a}^{t} e^{-\int_{a}^{r} h(s) d s} d r
$$

and accordingly

$$
v^{\prime}(t)=e^{-\int_{a}^{t} h(s) d s} .
$$


It is easy to see that

$$
v^{\prime \prime}(t)+h(t) v^{\prime}(t)=0, \quad t \in(a, c(\alpha)) .
$$

Differentiating $Q(t, \alpha)$ with respect to $t$, we get

$$
Q^{\prime}(t, \alpha)=u^{\prime}(t, \alpha)-\frac{v(t)}{v^{\prime}(t)} f(t, u(t, \alpha))
$$

and

$$
Q^{\prime \prime}(t, \alpha)=\left(-h(t)-\frac{v}{v^{\prime}} f_{u}(t, u)\right) u^{\prime}-\left(2+h(t) \frac{v(t)}{v^{\prime}(t)}\right) f(t, u)-\frac{v}{v^{\prime}} f_{t}(t, u) .
$$

Hence, we have

$$
\begin{aligned}
L(Q(t, \alpha)) & =Q^{\prime \prime}(t, \alpha)+h(t) Q^{\prime}(t, \alpha)+f_{u}(t, u) Q(t, \alpha) \\
& =-2\left(1+h(t) \frac{v(t)}{v^{\prime}(t)}\right) f(t, u)-\frac{v}{v^{\prime}} f_{t}(t, u) .
\end{aligned}
$$

From hypotheses $(F 2)$, we obtain

$$
L(Q(t, \alpha)) \leq 0, \quad t \in(a, c(\alpha)) .
$$

Since $\mathrm{Q}(t, \alpha)>0$ in $t \in(a, c(\alpha))$ and inequality (3.3) holds, by the Sturm comparison principle (see [2]), we see that $Q(t, \alpha)$ oscillates faster that $\varphi(t, \alpha)$. Hence, $\varphi(t, \alpha)$ has no zero in $t \in(a, c(\alpha))$. From $\varphi(a, \alpha)=0$ and $\varphi^{\prime}(a, \alpha)=1$, it follows that $\varphi(t, \alpha)>0$ for all $t \in(a, c(\alpha))$. The proof is complete.

Remark 3.1 Lemma 3.1 was already proved in [11]. Here we give a simpler proof, directly using Sturm comparison principle.

Now, we present a lemma which has been given to the case $g(|x|)=0$ (see [8]). To make the article as self-contained as possible, we will give a simple proof with a slight modification to [8].

Lemma 3.2 Assume $\alpha \in N$ and $f(t, u)$ satisfies ( $F 1)$, then

$(H 1) \varphi(t, \alpha)$ vanishes at least once and at most finitely many times in $(a, b(\alpha))$,

(H2) if $0<\alpha_{1}<\alpha_{2}$, and at least one of $u\left(t, \alpha_{1}\right)$ and $u\left(t, \alpha_{2}\right)$ has a finite zero, then they intersect in $\left(a, \min \left\{b\left(\alpha_{1}\right), b\left(\alpha_{2}\right)\right\}\right)$.

Proof. We shall prove this by contradiction. Suppose to the contrary that $\varphi(t, \alpha)$ does not vanish in $(a, b(\alpha))$, then $\varphi(t, \alpha)>0, t \in(a, b(\alpha))$. Note that $L(\varphi(t, \alpha))=0$, so we have

$$
\left(e^{\int_{a}^{t} h(s) d s} \phi^{\prime}(t, \alpha)\right)^{\prime}=-e^{\int_{a}^{t} h(s) d s} f_{u}(t, u(t, \alpha)) \phi(t, \alpha) .
$$

Using the definition of $L$, we have

$$
L(u(t, \alpha))=u^{\prime \prime}(t, \alpha)+h(t) u^{\prime}(t, \alpha)+f_{u}(t, u) u(t, \alpha) .
$$

Similar to (3.4), we have

$$
\left(e^{\int_{a}^{t} h(s) d s} u^{\prime}(t, \alpha)\right)^{\prime}=e^{\int_{a}^{t} h(s) d s} L(u(t, \alpha))-e^{\int_{a}^{t} h(s) d s} f_{u}(t, u(t, \alpha)) u(t, \alpha) .
$$

Multiply both sides of (3.4) by $u(t, \alpha)$ and (3.5) by $\varphi(t, \alpha)$, then subtract the resulting identities and we have 


$$
\begin{gathered}
\left(e^{\int_{a}^{t} h(s) d s}\left(\phi(t, \alpha) u^{\prime}(t, \alpha)-\phi^{\prime}(t, \alpha) u(t, \alpha)\right)\right)^{\prime} \\
=e^{\int_{a}^{t} h(s) d s}\left(u f_{u}(t, \alpha)-f(t, u)\right) \phi(t, \alpha) .
\end{gathered}
$$

By $(F 1)$, we have the right side of (3.6) is positive in $(a, b(\alpha))$. The left side of (3.6) is then a strictly increasing function of $t$ in (3.6). We get

$$
e^{\int_{a}^{t} h(s) d s}\left(\phi(t, \alpha) u^{\prime}(t, \alpha)-\phi^{\prime}(t, \alpha) u(t, \alpha)\right)>0 \quad \text { at } t=b(\alpha) .
$$

Thus, $e^{\int_{a}^{b(\alpha)} h(s) d s} \phi(b(\alpha), \alpha) u^{\prime}(b(\alpha), \alpha)>0$. However, it contradicts $u^{\prime}(b(\alpha), \alpha)<0$ and $\varphi(b(\alpha), \alpha) \geq 0$.

Since the rest of proof can be completed by the same argument as [8], we omit them.

Lemma 3.3 If (F1) and (F3) hold, then $\varphi(b(\alpha), \alpha) \neq 0$.

Proof. We shall prove this by contradiction. Suppose to the contrary that $\varphi(b(\alpha), \alpha)$ $=0$. Now, we may as well define $\tau(\alpha)$ to be the last zero of $\varphi(t, \alpha)$ in $(a, b(\alpha))$. By Lemma 3.1, it is easy to get $c(\alpha) \leq \tau(\alpha)$, thus $u^{\prime}(\tau(\alpha), \alpha) \leq 0$ and $u^{\prime}(t, \alpha)<0$ for all $t \in$ $(\tau(\alpha), b(\alpha)]$. We introduce a function

$$
G(t, \alpha)=u^{\prime}(t, \alpha)
$$

Differentiating $G(t, \alpha)$ with respect to $t$, we get

$$
G^{\prime}(t, \alpha)=u^{\prime \prime}(t, \alpha)=-h(t) u^{\prime}-f(t, u)
$$

and

$$
G^{\prime \prime}(t, \alpha)=-h^{\prime}(t) u^{\prime}(t, \alpha)-h(t) u^{\prime \prime}(t, \alpha)-f_{u}(t, u) u^{\prime}-f_{t}(t, u) .
$$

Hence,

$$
\begin{aligned}
L(G(t, \alpha)) & =G^{\prime \prime}(t, \alpha)+h(t) G^{\prime}(t, \alpha)+f_{u}(t, u) G(t, \alpha) \\
& =-h^{\prime}(t) u^{\prime}(t, \alpha)-h(t) u^{\prime \prime}(t, \alpha)-f_{u}(t, u) u^{\prime}(t, \alpha)-f_{t}(t, u) \\
& -h^{2}(t) u^{\prime}(t, \alpha)-h(t) f(t, u)+f_{u}(t, u) u^{\prime}(t, \alpha) \\
& =-h^{\prime}(t) u^{\prime}(t, \alpha)+f_{t}(t, u) .
\end{aligned}
$$

Hence, we have

$$
\left(e^{\int_{a}^{t} h(s) d s} G^{\prime}(t, \alpha)\right)^{\prime}=e^{\int_{a}^{t} h(s) d s} L(G(t, \alpha))-e^{\int_{a}^{t} h(s) d s} f_{u}(t, u(t, \alpha)) G(t, \alpha) .
$$

Similar to the argument of Lemma 3.2, multiply both sides of (3.4) by $G(t$, $\alpha$ ), and (3.7) by $\varphi(t, \alpha)$ then we have

$$
\left(e^{\int_{a}^{t} h(s) d s}\left(\phi(t, \alpha) G^{\prime}(t, \alpha)-\phi^{\prime}(t, \alpha) G(t, \alpha)\right)\right)^{\prime}=e^{\int_{a}^{t} h(s) d s} L(G(t, \alpha)) \phi(t, \alpha) .
$$

Note that $\varphi(b(\alpha), \alpha)=0$, thus integrating both sides of (3.8) from $\tau(\alpha)$ to $b(\alpha)$, we obtain

$$
\begin{aligned}
& \left(-e^{\int_{a}^{b(\alpha)} h(s) d s} \phi^{\prime}(b(\alpha), \alpha) G(b(\alpha), \alpha)\right)-\left(-e^{\int_{a}^{\tau(\alpha)} h(s) d s} \phi^{\prime}(\tau(\alpha), \alpha) G(\tau(\alpha), \alpha)\right) \\
& =\int_{\tau(\alpha)}^{b(\alpha)} e^{\int_{a}^{t} h(s) d s} L(G(t, \alpha)) \phi(t, \alpha) d t .
\end{aligned}
$$


Since $\tau(\alpha)$ to be the last zero of $\varphi(t, \alpha)$ in $(a, b(\alpha))$, the behavior of $\varphi(t, \alpha)$ in $(\tau(\alpha), b$ $(\alpha))$ can be classified into two cases as follows:

(i) $\varphi(t, \alpha)>0$ in $(\tau(\alpha), b(\alpha))$, then the left side of (3.9) is negative, but by (F3) the right side is positive.

It is impossible.

(ii) $\varphi(t, \alpha)<0$ in $(\tau(\alpha), b(\alpha))$, then the left side of (3.9) is positive, but by (F3) the right side is negative.

It is also impossible. The proof is complete.

The proof of Theorem 3.1 We will prove it as a standard process. Assume that $N$ is a nonempty set, otherwise we have nothing to prove. Let $\alpha \in N$, then $u(b(\alpha), \alpha)=0$. It is easy to see that $u^{\prime}(b(\alpha), \alpha) \leq 0$. If $u^{\prime}(b(\alpha), \alpha)=0$, then the assumption $f(t, 0) \equiv 0$ for all $t \geq 0$, and the uniqueness of solution of initial value problems for ordinary differential equations imply that $u(t, \alpha) \equiv 0$ for all $t \in[a, b(\alpha)]$, which contradicts the initial condition of $u(t, \alpha)$. Hence, we have

$$
u^{\prime}(b(\alpha), \alpha)<0,
$$

and the implicit function theorem implies that $b(\alpha)$ is well-defined as a function of $\alpha$ in $N$ and $b(\alpha) \in C^{1}(N)$. Furthermore, it follows from (3.10) that $N$ is an open set. By Lemma 3.2, we have $N$ is an open interval (see [8]).

Differentiate both sides of the identity $u(b(\alpha), \alpha)=0$ with respect to $\alpha$, we obtain

$$
u^{\prime}(b(\alpha), \alpha) b^{\prime}(\alpha)+\phi(b(\alpha), \alpha)=0 .
$$

From above Lemma 3.3, we have $\varphi(b(\alpha), \alpha) \neq 0$. Thus, $b^{\prime}(\alpha) \neq 0, \alpha \in N$. It means that $b^{\prime}(\alpha)$ does not change sign, i.e., $b(\alpha)$ is monotone. The proof is complete.

Remark 3.2 Actually, if the functions $f(|x|, u)$ and $g(|x|)$ satisfy some suitable conditions, it is not difficult to get the existence of positive radial solutions to the Dirichlet boundary value problem (1.1). We just need that for Equation 2.1, the functions $f(|x|$, $u)$ and $g(|x|)$ satisfy inequality (2.2) and

$$
\int_{a}^{b} e^{-\int_{a}^{s} h(\xi) d \xi}\left(\alpha-\int_{a}^{s} e^{\int_{a}^{\xi} h(r) d r} f(\xi, u) d \xi\right) d s=0 .
$$

However, it seems that these assumptions are too strict.

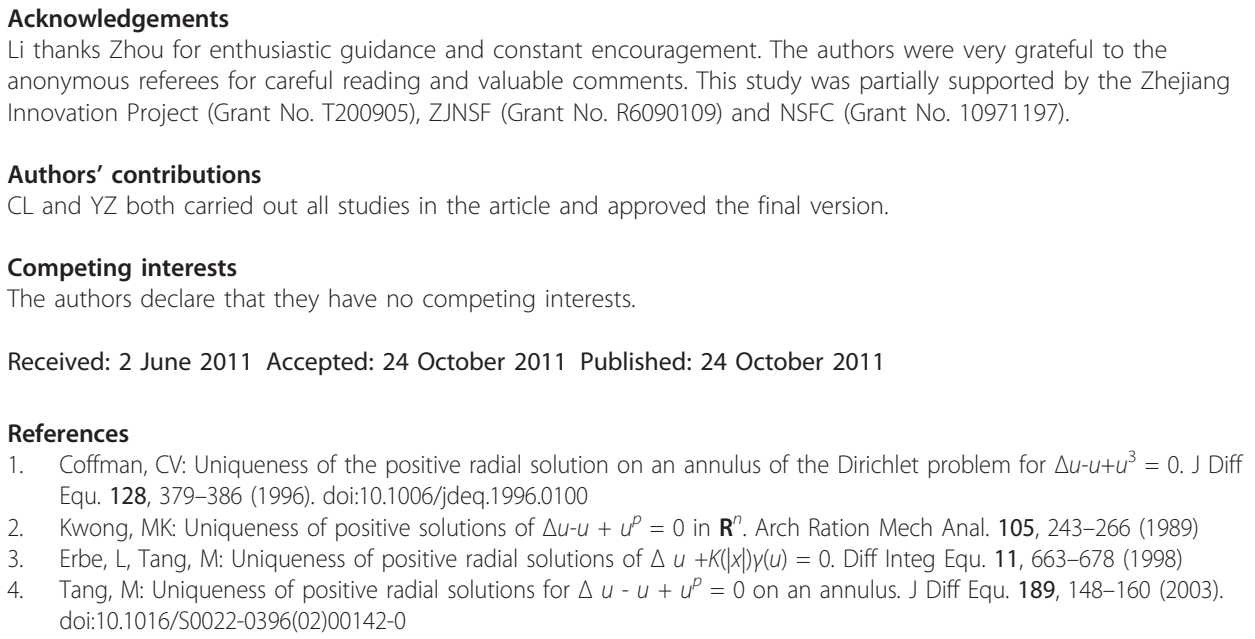


5. Felmer, $P$, Martínez, $S$, Tanaka, K: Uniqueness of radially symmetric positive solutions for $-\Delta u+u=u^{p}$ in an annulus. J Diff Equ. 245, 1198-1209 (2008). doi:10.1016/j.jde.2008.06.006

6. Korman, P: Uniqueness and exact multiplicity of solutions for a class of Dirichlet problems. J Diff Equ. 244, 2602-2613 (2008). doi:10.1016/j.jde.2008.02.014

7. $\mathrm{Ni}, \mathrm{WM}$, Nussbaum, RD: Uniqueness and nonuniqueness for positive radial solutions of $\Delta u+f(u, r)=0$. Commun Pure Appl Math. 38, 67-108 (1985). doi:10.1002/cpa.3160380105

8. Erbe, L, Tang, M: Uniqueness of positive radial solutions of $\Delta u+f(|x| u)=0$. Diff Integ Equ. 11, 725-743 (1998)

9. Conway, JB: A Course in Function Analysis. Springer, New York (1990)

10. Franchi, B, Lanconelli, E, Serrin, J: Existence and uniqueness of nonnegative solutions of quasilinear equations in $\mathbf{R}^{n}$. Adv Math. 118, 177-243 (1996). doi:10.1006/aima.1996.0021

11. Ma, R, An, Y: Uniqueness of positive solutions of a class of O.D.E. with Robin boundary conditions. Nonlinear Anal. 63, 273-281 (2005). doi:10.1016/j.na.2005.05.012

doi:10.1186/1687-2770-2011-38

Cite this article as: Li and Zhou: Uniqueness of positive solutions to a class of semilinear elliptic equations. Boundary Value Problems 2011 2011:38.

\section{Submit your manuscript to a SpringerOpen ${ }^{\circ}$} journal and benefit from:

- Convenient online submission

- Rigorous peer review

- Immediate publication on acceptance

- Open access: articles freely available online

- High visibility within the field

- Retaining the copyright to your article

Submit your next manuscript at $\gg$ springeropen.com 